\title{
Experimental study on transient behavior of embedded spirall-coil heat exchanger
}

\author{
E. I. Jassim \\ Department of Mechanical Engineering, Prince Mohammad Bin Fahd University, PMU, \\ Al-Khobar, Saudi Arabia \\ Correspondence to: E. I. Jassim (ejassim@pmu.edu.sa)
}

Received: 9 April 2015 - Accepted: 5 September 2015 - Published: 11 September 2015

\begin{abstract}
Spiral coil offers a substantial amount of heat transfer area at a considerably low cost as it does not only have a lower wall resistance but it also achieves a better heat transfer rate in comparison to conventional $U$ tube arrangement. The general aim of the study is to assess different configurations of spiral coil heat exchangers that can eventually operate in a highly efficient manner.

The paper documents the transient behavior of spiral-shaped tubes when the coil is embedded in a rectangular conducting slab. Different arrangements and number of turns per unit length, with fixed volumes, are considered in order to figure out the optimal configuration that maximizes the performance of the heat transfer. The implementation presented in the study is conducted to demonstrate the viability of the use of a large conducting body as supplemental heat storage.

The system uses flowing water in the coil and stagnant water in the container. The copper-made coils situated in the center of the slab carries the cold fluid while the container fluid acts as a storage-medium. The water temperature at several depths of the container was measured to ensure uniformity in the temperature distribution of the container medium.

Results have shown that the coil orientation, the number of loops, and the Reynolds number, substantially influence the rate of the heat transfer. The vertically-embedded spiral coil has a better performance than the horizontally-embedded spiral coil. Doubling the number of loops is shown to enhance the performance of the coil. Increasing Reynolds Number leads to better coil performance.
\end{abstract}

\section{Introduction}

For almost a century after the introduction of the curved tubes heat exchanger, much attention have recently been drawn towards the enhancement techniques in energy and the mass transfer of spiral coil heat exchanger. The popularity of the spiral coil heat exchanger is coming from its wide applications, due to usefulness and suitability, as it proves to be compact in design, unique in geometrical structure, and feasible in manufacturing. However, these benefits are not the only reasons behind the attraction of this type of heat exchanger. The eminence of such system over the straight tube type, in terms of heat and mass transfer, gives them domination over the conventional type thermal system. Today, with the advancements in science and technology, spiral tube heat exchangers have become prevalent in numerous thermal applications globally.

The primary reason of such superiority is attributed to the motion induced by the coil curvature, as it relies on the dimensionless number named as the Dean Number, defined as the ratio of centrifugal force to inertial force. It can be expressed as:

$D e=R e \sqrt{\frac{d_{\mathrm{p}}}{2 R}}$

where $d_{\mathrm{p}}$ is the pipe diameter, and $R$ is the radius of curvature of the coil.

W. R. Dean first induced Dean Vortices during his study on how heat transfer can be enhanced in spiral piping systems 
(Dean, 1927a, b). The Dean vortex considers several important factors, such as curvature ratio, viscosity, and Reynolds number. The Dean Vortices method has rarely been examined in regards to the study of the heat transfer enhancement of spiral piping. Nevertheless, recent research on enhancement of heat transfer in the spiral piping has been conducted using the Dean Vortices method. Recent studies and investigations have proved that the thermal coupling between spiral pipes and heat transfer depends, to a great extent, on Dean Vortex's motion (Elazm et al., 2013).

Dean Vortices also influence the flow's critical Reynolds number. The critical Reynolds Number that describes the transition of the flow inside coiled tubes from laminar to turbulent is much higher than that for conventional straight tubes heat exchanger. Dean Vortex tends to restrict turbulence inception, delaying the flow transition to the turbulent. Hence, it should be considered together with the Reynolds Number in describing the flow regime. Srinivasan et al. (1970) came up with a correlation of the critical Reynolds number as a function of the coil geometry:

$R e_{\mathrm{c}}=2100\left(1+12 \sqrt{\frac{d_{\mathrm{p}}}{2 R}}\right)$.

The design criteria of the spiral tube heat exchanger vary with the intended functionality (Sanner et al., 2003). For instance, in geothermal application, heat exchangers are available in two types: open, and closed type heat exchangers. The former is a direct system between the ground and the system. The latter, however, is an indirect type of system in which the pipes are buried in vertical or horizontal patterns. The condition of the nearby fluid determines the suitability and appropriateness of vertical versus horizontal ground heat exchangers (Alalaimi et al., 2014).

Temperature complexities that are caused by curvaturebased torsion arise a unique advantage to the process. It can only be observed in the case of spiral pipes (Sasmito et al., 2011). Consequently, much research study and implementation of spiral pipe heat transfer exchangers are nowadays demanded for various applications.

The spiral piping heat-coupling mechanism is attributed to many factors, such as geometrical configurations, compact size, bigger thermal conduction space, number of loops, etc. Numerous studies have been conducted to investigate the configuration and geometry of spiral pipes that could enhance the thermal coupling between them and the conducting volume. Heo and Chung (2012) have described thermal coupling using spiral piping with various shapes and sizes, such as the length of the coil, the number of turns, the coil radius, and the helix diameter. The natural transfer of thermal energy was also studied under different conditions, including the spiral pipe dimensions, rate of flow of the fluid, the bath temperature, and the inlet temperature (Heo and Chung, 2012). He proved that the spiral shape is more effective than the $U$-shape in terms of the rate of heat transfer.
Other studies conducted by different researchers have emphasized the fact that spiral tubes are indeed better than any other form of tubes, as they effectively act as heat transfer enhancers. Therefore, despite the differences of studies on spiral pipe usage in industrial applications, the outcome results of both experimental and analytical studies are the same.

Jamshidi et al. (2013) experimentally analyzed the enhancement of heat transfer for the coiled tube heat exchanger. The parameters of interest set in their study were: tube diameter, coil curvature, number of loops, coil pitch, and shell and tube side flow rate. They utilized the Wilson plot and the Taguchi technique during the tests. The shell side Reynolds number was found to be the primary factor for the reduction in thermal resistance. However, Zhu et al. (2014) reported that the Nusselt number of the flow inside the spiral tubes increase with the Reynolds number.

Heat transfer by natural convection has been experimentally studied by Ali (2006) using vertical coil at a range of Prandtl number 250-400. Different configurations of the helix-to-tube diameter ratio $30,20.83,17.5,13.33$, and 10 were employed. Three empirical correlations based on characteristics length were developed for: $250<\operatorname{Pr}<400$.

$$
\begin{array}{ll}
N u_{\mathrm{L}}=0.619 R a_{\mathrm{L}}^{0.3} & \text { valid for } 4.37 \times 10^{10} \leq R a_{\mathrm{L}} \leq 5.5 \times 10^{14} \\
N u_{\mathrm{L}}=0.555 G r_{\mathrm{L}}^{0.301} \operatorname{Pr}^{0.314} & \text { valid for } 1 \times 10^{8} \leq G r_{\mathrm{L}} \leq 5.0 \times 10^{14} \text { and } 4.4 \leq \operatorname{Pr} \leq 345 \\
N u_{\mathrm{L}}=0.714 R a_{\mathrm{L}}^{0.294} & \text { valid for } 4.35 \times 10^{10} \leq R a_{\mathrm{L}} \leq 8.0 \times 10^{14}
\end{array}
$$

The impact of curvature ratio was also addressed by Beigzadeh and Rahimi (2012) using artificial neural networks (ANNs). The characteristics of the flow, including heat transfer, were of interest.

Experimental tests on spiral coil heat exchanger for boiling purposes has been reported by Elsayed et al. (2012). Effects of tube and helix diameters on the heat transfer coefficients were investigated using different sizes. It was observed that an enhancement of up to $63 \%$ was obtained in the heat transfer coefficient, when the smaller tube diameter was employed. A similar conclusion was reported by Gupta et al. (2011). Observing from the experiments on different tube sizes, they found that a smaller size tube enhances the performance of the coil by increasing the rate of heat transfer. Decreasing the helix diameter of the coil could also improve the heat transfer coefficient up to a level of $150 \%$ (Elsayed et al., 2012).

Freng et al. (2012) numerically studied the thermo-fluid features of the spiral coil heat exchanger at a different Dean Number and pitch size. Previous experimental works validated the CFD methodology. The Nusselt number increases as the pitch size increases. However, as long as the curvature ratio remains constant, no alteration in the behavior of the Nusselt Number was observed when the torsion ratio is changed (Freng et al., 2012).

Recently Seara et al. (2014) developed a numerical model to illustrate the thermal and hydraulic characteristics of the vertical spiral heat exchanger. They validated the numerical 
outcomes with experimental data obtained from lab facilities under various operating conditions. Among several different correlations, the following expression has showed good agreement with the experimental results.

$N u_{\mathrm{do}}=0.499 R a_{\mathrm{do}}^{0.2633}$ for $4.67 \times 10^{6} \leq R a_{\mathrm{do}} \leq 3.54 \times 10^{7}$

Spiral coils buried in conducting volumes have scarcely been addressed in the literatures. Applications of such a type heat exchanger include: geothermal heat pumps, extracting oil and gas, and other solid-fluid interaction applications. Despite several numerical, analytical, and experimental papers that have been published to address the heat transfer mode of embedded coil, limited studies have been conducted to optimize the configuration and orientation of the coil, as well as the heat transfer mechanism during the transient process. Parameters such as number of loops per unit tube length, mass flow rate, coil orientation, and coil pitch are some of the many factors that influence the heat transfer efficiency. Yet, further emphasis is required to address their effects on coil performance and heat transfer rate. The main objectives of this paper include studying and experimentally analyzing the behavior of spiral coil embedded in a conducting container, highlighting the impact of orientation and number of loops on the coil performance, as well as presenting the spatial and temporal mechanisms of the heat transfer process.

\section{Theories and assumptions}

\subsection{The 1-D unsteady state energy analysis}

Consider a conducting volume that contains a medium (water) of mass $M$, volume $V$, density $\rho$, and specific heat $C_{\mathrm{p}}$, at a uniform initial temperature $T_{\text {wi }}$. At time $t=0$, a coiled-tube pipe with surface area $A_{\mathrm{s}}$, carrying a fluid of mass flow $\dot{m}_{1}$, is sunk into the medium. Energy transfer occurs between the medium in the container and the fluid inside the coil by natural convection $(h)$. For simplicity, we assume that the transient temperature of the medium $T(t)$ is less than its initial temperature $T_{\text {wi }}$. However, the energy analysis could also be used for the opposite case. We also assume that the medium inside the container has a uniform instantaneous temperature that only changes with time, $T=T(t)$. The analysis ignores the coil thermal resistance since it is comprised of a high conductive material (copper). Copper thermal resistance is of order $10^{-2}$ of convective heat transfer. The error in the convective heat transfer coefficient of the coil side was not exceeding $1 \%$.

The temperature of the medium drops by a differential value $\mathrm{d} T$ for a differential time interval $\mathrm{d} t$.

The energy balance of the system for the time interval $\mathrm{d} t$ can be expressed as:

$$
\begin{aligned}
M \times C_{\mathrm{p}} \times \frac{\mathrm{d} T}{\mathrm{~d} t} & =h \times A_{\mathrm{s}} \times\left(T-T_{\mathrm{s}}\right) \frac{\mathrm{d}\left(T-T_{\mathrm{s}}\right)}{T-T_{\mathrm{s}}} \\
& =\frac{h \times A_{\mathrm{S}}}{\rho \times V \times C_{\mathrm{p}}} \times \mathrm{d} t \text { or } \frac{T(t)-T_{\mathrm{s}}}{T_{\mathrm{wi}}-T_{\mathrm{s}}} \\
& =e^{-B \times t} \text { where } B=\frac{h A_{\mathrm{s}}}{\rho V C_{\mathrm{p}}} .
\end{aligned}
$$

The surface area of the coil will be compared using two scenarios. The first scenario ignores the effect of the curvature and assumes the 1-D conventional tube surface area is applied; i.e. $\left(A_{\mathrm{s}}=\pi d_{\mathrm{p}} l\right)$, where $l$ is the coil length. In contrast, the second scenario considers the coil geometry by introducing the total outside heat transfer area $\left(A_{\text {coil }}\right)$ for spiral coil. The area can be obtained from the following expression (Wijeysundera et al., 1996):

$A_{\mathrm{coil}}=\pi D A_{\mathrm{o}} n(1+2 \pi \alpha n / D)$

where $A_{0}$ stands for the heat transfer area per unit length $\left(\pi d_{\mathrm{p}}\right) ; n$ is the number of turns; $D$ is the helix diameter; and $\alpha$ is the rate of increase of the helix radius, in $\left(\mathrm{m} \mathrm{rad}^{-1}\right)$.

The external natural convective heat transfer coefficient $(h)$ for each time step can be obtained from the following expressions (Yunus and Afshin, 2010):

$$
\begin{aligned}
& N u=\left\{0.825+\frac{0.387 R a_{\mathrm{L}}^{1 / 6}}{\left[1+(0.492 / P r)^{9 / 16}\right]^{8 / 27}}\right\}^{2} \text { for verticle coil } \\
& N u=\left\{0.6+\frac{0.387 R a_{\mathrm{D}}^{1 / 6}}{\left[1+(0.599 /)^{9 / 16}\right]^{8 / 27}}\right\}^{2} \text { for horizontal coil }
\end{aligned}
$$

where $N u=\frac{h L_{\mathrm{c}}}{k}$; for vertical position and $N u=\frac{h D}{k}$; for horizontal position

$R a=G r_{\mathrm{Lc}} \operatorname{Pr}=\frac{g \beta\left(T(t)-T_{\mathrm{s}}\right) L_{\mathrm{c}}^{3}}{v^{2}} \operatorname{Pr} L_{\mathrm{c}}= \begin{cases}H_{\mathrm{t}} & \text { height of the coil for veritcal position } \\ D & \text { Helix diameter for horizontal position }\end{cases}$

Helix diameter is obtained from the following expression (Ali, 2006):

$D=\frac{\sum_{i=1}^{N} \sqrt{D_{\mathrm{si}}^{2}-\left(\frac{H_{\mathrm{i}}}{\pi}\right)^{2}}}{N}$

where $D_{\text {si }}$ is the slanted outer turn diameter for each loop (Fig. 1b).

The transient surface temperature of the spiral coil can be obtained by employing Eq. (5).

\subsection{The coil inlet and exit temperatures}

The temperature sensors at inlet and outlet of the pipe are mounted outside the conducting volume, as seen in Fig. 2. Since the coil has an extended straight portion at its ends, it is necessary to predict the coil inlet and exit temperatures, $\left(T_{\mathrm{ci}}\right)$ and $\left(T_{\mathrm{ce}}\right)$, from the measured inlet and exit temperatures, $\left(T_{\mathrm{i}}\right)$ and $\left(T_{\mathrm{e}}\right)$, respectively. The following expressions 


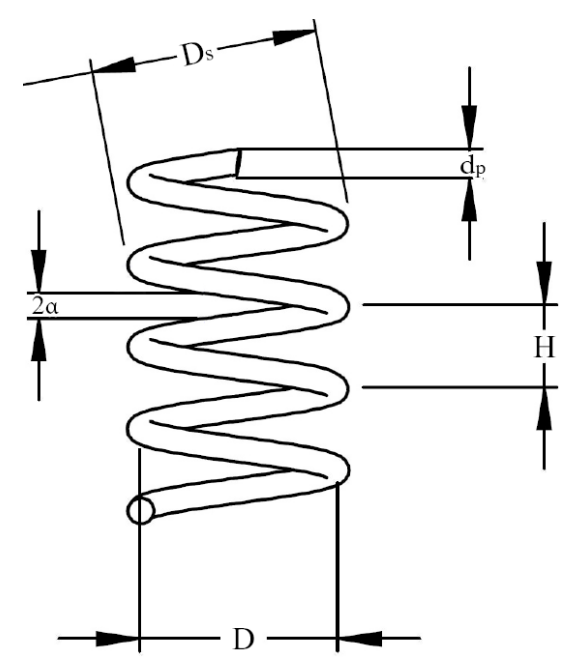

(a)

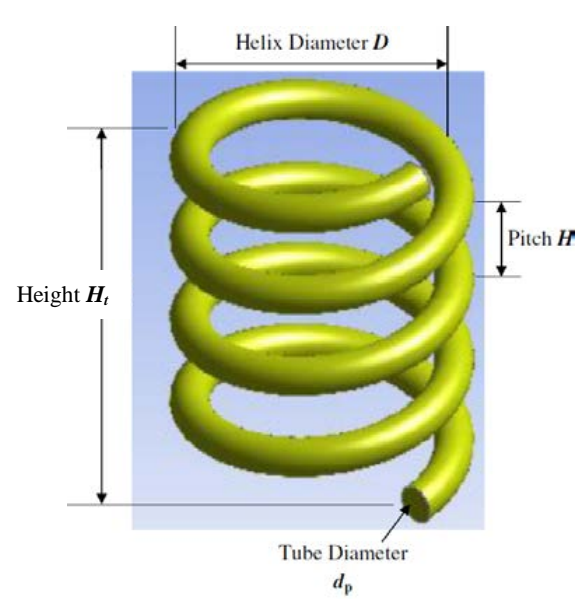

(b)

Figure 1. Typical geometry of spiral coil: (a) plane view and (b) isometric.

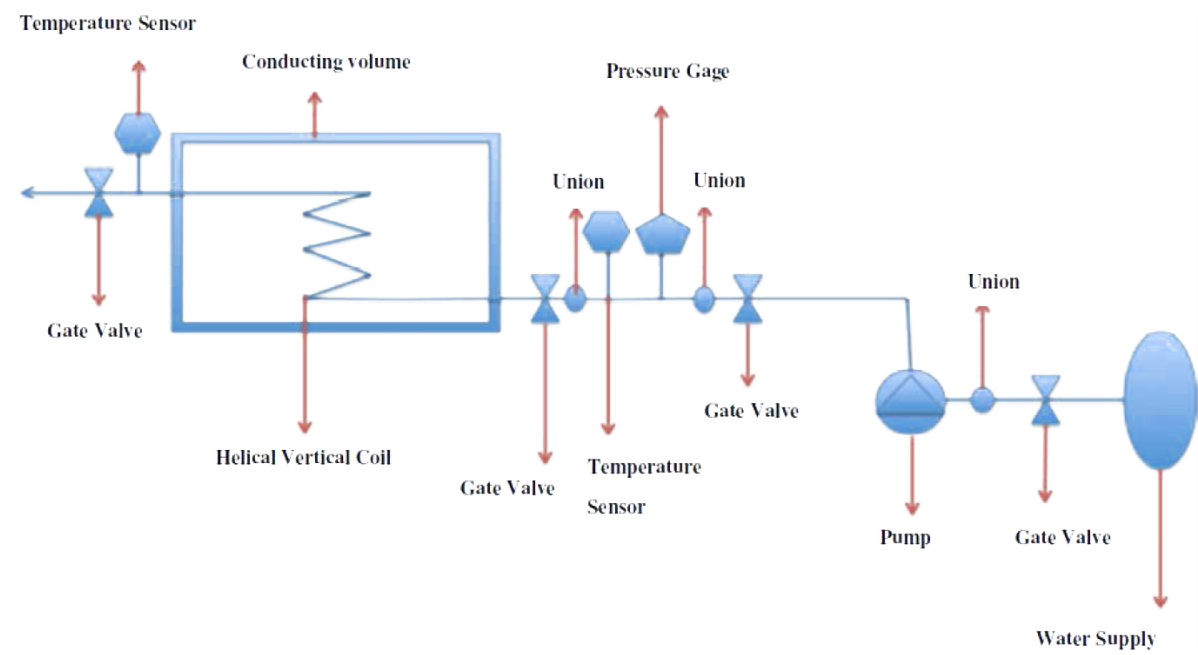

Figure 2. Experiment apparatus.

have been derived (see Appendix A) and employed to determine the fluid temperatures at the coil's ends.

$T_{\mathrm{ci}}=T_{\mathrm{i}}\left(\frac{1-0.5 \mathrm{NTU}}{1+0.5 \mathrm{NTU}}\right)+T(t) \times\left(\frac{\mathrm{NTU}}{1+0.5 \mathrm{NTU}}\right)$

$T_{\mathrm{ce}}=T_{\mathrm{e}}\left(\frac{1+0.5 \mathrm{NTU}}{1-0.5 \mathrm{NTU}}\right)-T(t) \times\left(\frac{\mathrm{NTU}}{1-0.5 \mathrm{NTU}}\right)$

For convenience, the temperature difference across the straight portion of the coil was calculated at the entrance and exit regions (see Appendix A). It has concluded that not more than $1 \%$ of the increment in temperature has been gained across the uncoiled pipe while $99 \%$ of the temperature rise occurs across the spiral coil. Hence, it is reasonable to assume that the measured inlet and exit temperatures are equal to their corresponding inlet and exit temperatures of the helical part.

\section{Experimental set-up}

For the purpose of the experiment, copper-tube coils with a diameter of $19 \mathrm{~mm}$ and a length of $10.6 \mathrm{~m}$ are used. Only $5 \%$ of the length is straight before and after the helical portion. The tubes are carefully furnished from the inner surface to reduce flow resistance. External pipes are connected to the coil with straight joints at the two ends. Other than the container, insulation was applied to the external pipes to avoid any heat gain/loss from the surroundings. Thermal insulation material, wool blanket $\left(k=0.04 \mathrm{~W} \mathrm{~m}^{-1} \mathrm{~K}^{-1}\right)$, covers the conducting volume (container) to minimize the heat 


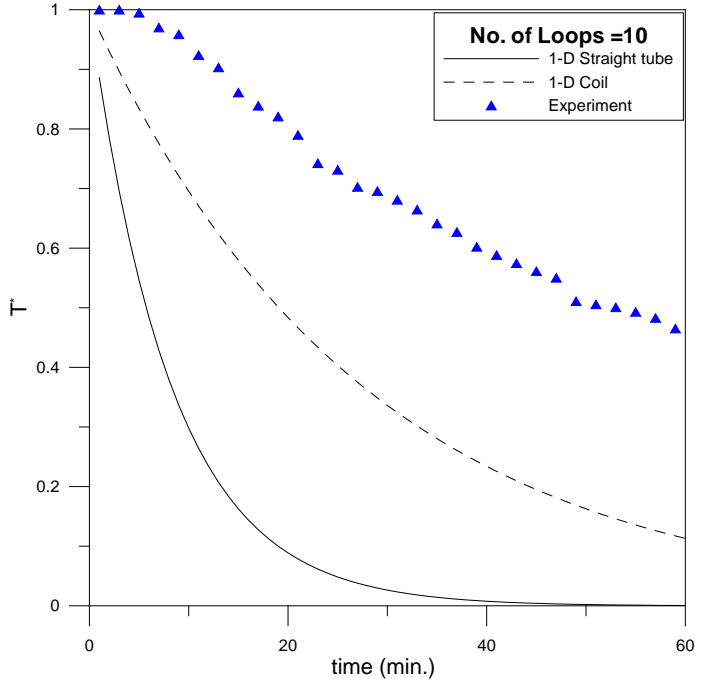

Figure 3. Impact of curvature on transient behavior of the coil.

losses to the surroundings. The heat lost by the container to the surrounding does not exceed $0.5 \%$ of the heat exchange between the coil and the water in the container. For such small amount of heat loss, the assumption of perfectly insulated system for the container is fair and reasonable.

Four electric heaters, each holding a capacity of $2500 \mathrm{~W}$, are mounted at the bottom of the container to provide heat energy to the water of the container. A thermal regulator is installed to stabilize the water pool temperature within a $\pm 0.1^{\circ} \mathrm{C}$ precision. The pump and the ball valve regulate the flow of fluids inside the spiral pipes. The ball valve is specifically designed to maintain a particular flow rate of fluid flowing within the coil when the pump is under operation. A centrifugal pump is connected to pump fluid through the spiral pipes at varying Reynolds numbers.

During the experiment, the fluid flowing inside the coil absorbs heat energy from the water of the container while passing through the spiral tube. The inlet/outlet temperatures of the spiral pipes are measured using the installed digital thermometers. Similarly, the inlet/outlet pressures were also measured using the installed pressure transducers. Important data were captured after every moment and recorded until a steady state occurs.

\section{Results and discussion}

Experiments are carried out for different boundary conditions and coil parameters, such as: coil pitch, number of loops, Reynolds number, and coil orientation. The constraints of the experiments are illustrated in Table 1.
Table 1. Thermal properties and system dimensions.

\begin{tabular}{lll}
\hline Property & Unit & Value \\
\hline Pipe diameter & $\mathrm{mm}$ & 19 \\
Coil length & $\mathrm{m}$ & 10.6 \\
Container $(l \times w \times h)$ & $\mathrm{m}$ & $1.14 \times 0.65 \times 0.48$ \\
Density & $\mathrm{kg} \mathrm{m}^{-3}$ & 994 \\
Dynamic viscosity & $\mathrm{kg} \mathrm{m}^{-1} \mathrm{~s}^{-1}$ & $0.653 \times 10^{-3}$ \\
Thermal conductivity & $\mathrm{W} \mathrm{m}^{-1} \mathrm{~K}^{-1}$ & 0.648 \\
Specific heat & $\mathrm{J} \mathrm{kg}^{-1} \mathrm{~K}^{-1}$ & 4190 \\
Prandtl number & & 4.23 \\
\hline
\end{tabular}

\subsection{Coil geometry}

The coil selected for studying the influence of its geometry has a helix diameter of $(D=15 \mathrm{~cm})$, a pitch of $(H=2 \mathrm{~cm})$, and 10 loops. The coil is firmly mounted vertically and sunk completely inside the pool. The coil inlet/exit temperatures and pressures, container medium temperature, and flow rate are recorded each minute.

A dimensionless temperature of the medium is plotted against time for a time span of an hour to ensure the occurrence of the steady state condition. Three curves are plotted: 1-D tube (ignoring the curvature of the coil), 1-D coil (including the curvature of the coil), and the experiment results. As seen in Fig. 3, the temperature of the medium in the container drops exponentially for all cases. However, the steep of the curves are not identical. In fact, the reduction in the medium temperature for the 1-D coil is not as sharp as the reduction for the 1-D tube. This could be attributed to the impact of coil geometry, which is excluded in the analysis of the 1-D tube, hence strengthening the essential demand of considering the coil configuration during the spiral coil design process. Although the results of the 1-D coiled-type analysis are somewhat acceptable, the experiment outcome is shown to be notably different since the Dean vortex is excluded in 1-D theory.

Figure 4 depicts the performance of the coil, Eq. (10), with time only for the case of experiment measurements. The coil efficiency asymptotically reaches its steady value (24\%) quickly, despite the process still being in the transient region.

$\eta=\frac{T_{\mathrm{ce}}(t)-T_{\mathrm{ci}}(t)}{T_{\mathrm{wi}}(t)-T_{\mathrm{ci}}(t)}$

\subsection{Number of loops}

Another coil of 5 loops with similar length and pitch, but a different loop diameter $(30 \mathrm{~cm})$, is vertically investigated under similar conditions that are illustrated in Table 1. The behavior of the coil, in correspondence with the change in the number of loops, is presented in Figs. 5-7. The comparison of the normalized temperature of the medium clearly portrays that increasing the number of loops also increases 


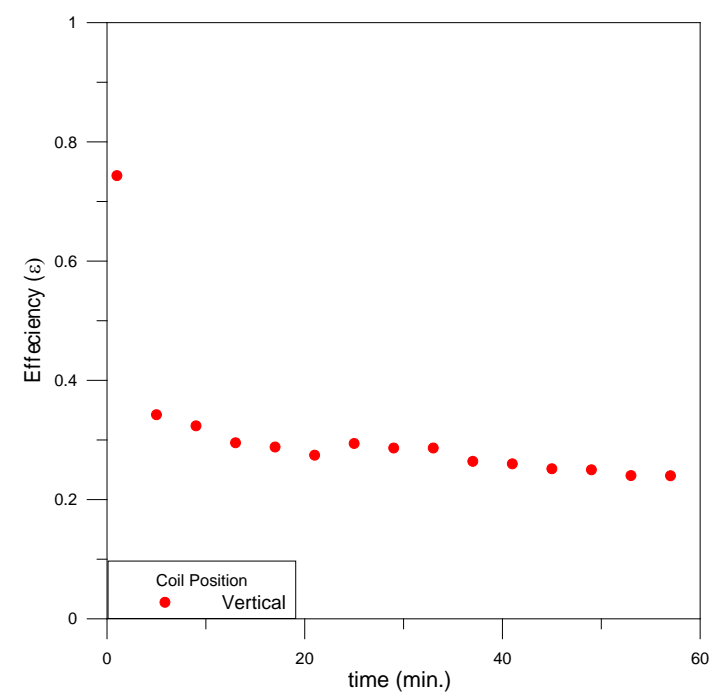

Figure 4. Efficiency variation of the coil with time.

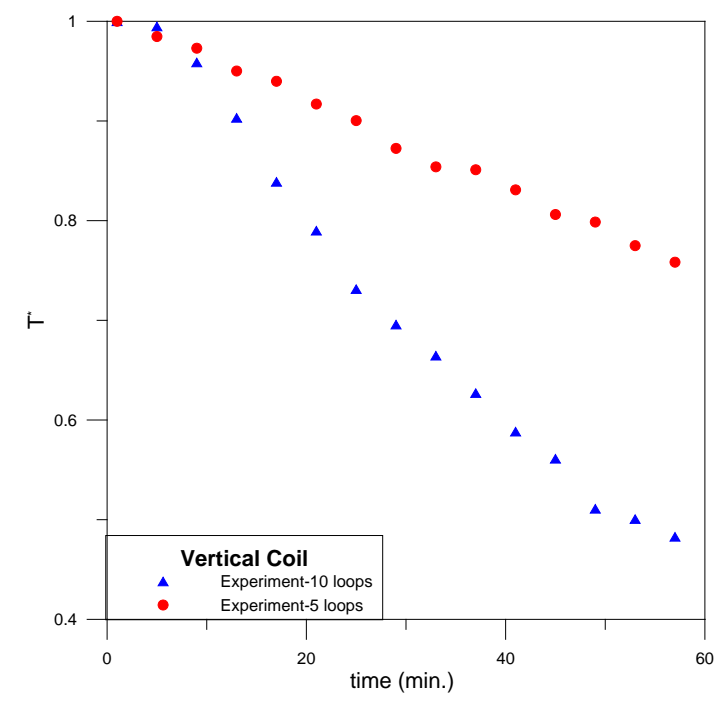

Figure 5. Influence of number of loops.

the rate of heat transfer, despite the length of the two coils being identical.

Figure 6 shows that disregarding the curvature of the coil leads to an insignificant impact of the loops on the coil performance. Hence, one would expect a remarkable error in the designed parameters during the process of analysis of the spiral coil heat exchanger.

Coil efficiency is expected to improve following the enhancement that occurred in the rate of the heat transfer, as depicted in Fig. 7.

\subsection{Coil orientation}

To investigate the impact of coil orientation, the 10-looped vertical coil is fabricated to sink horizontally in the container.

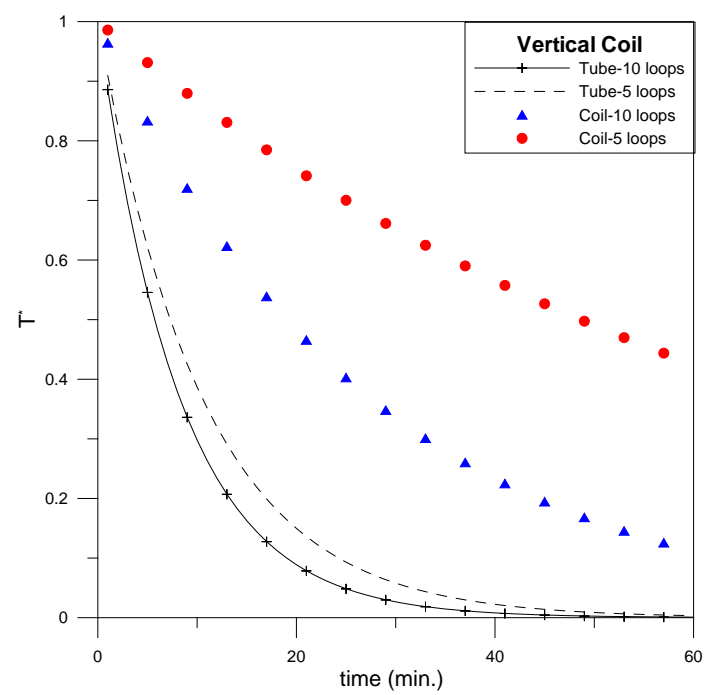

Figure 6. Comparison of 1-D analysis for straight tub vs. coil.

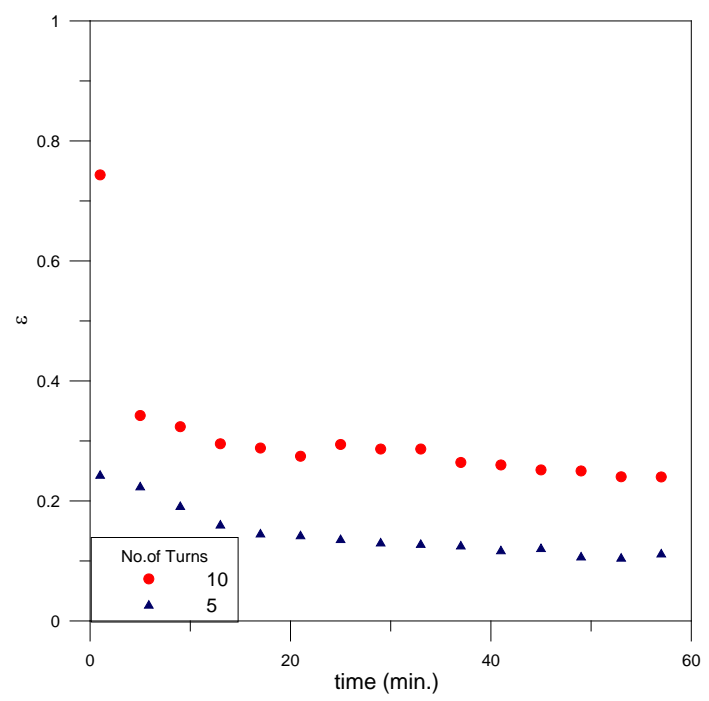

Figure 7. Effect of coil loops on the performance.

Figure 8 presents the normalized temperature variation with a time step for vertical and horizontal positions. Based on the experiment results, in terms of the rate of heat transfer, placing the coil vertically is shown to be better than placing the coil horizontally. However, no such outcome was obtained when 1-D analysis was applied, despite the coil curvature being considered. This important conclusion manifests the drawbacks of relying on the 1-D analysis of the coil and enforces the demand of experimental studies for obtaining reliable results.

The performance of the vertically-positioned coil is shown to be more enhanced than the horizontal position, as presented in Fig. 9. 


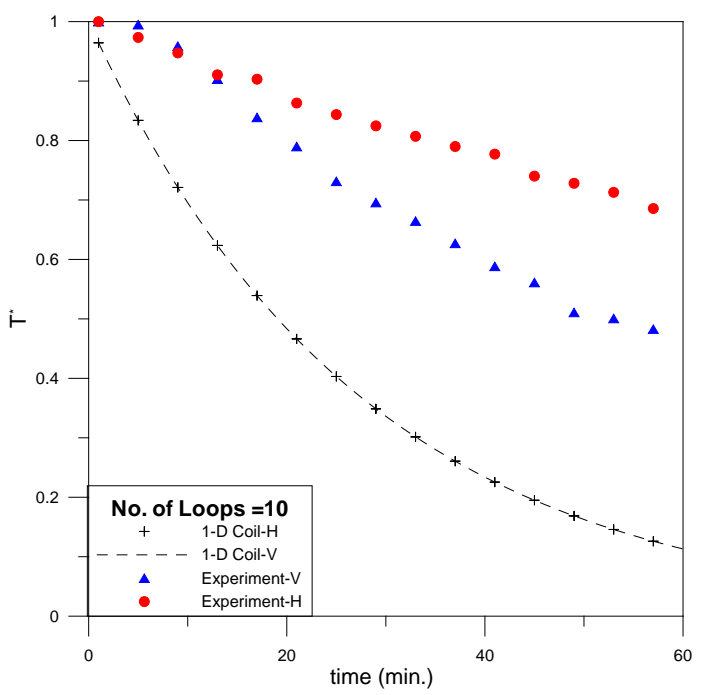

Figure 8. Temperature variation predicted by 1-D coiled-type and experiment.

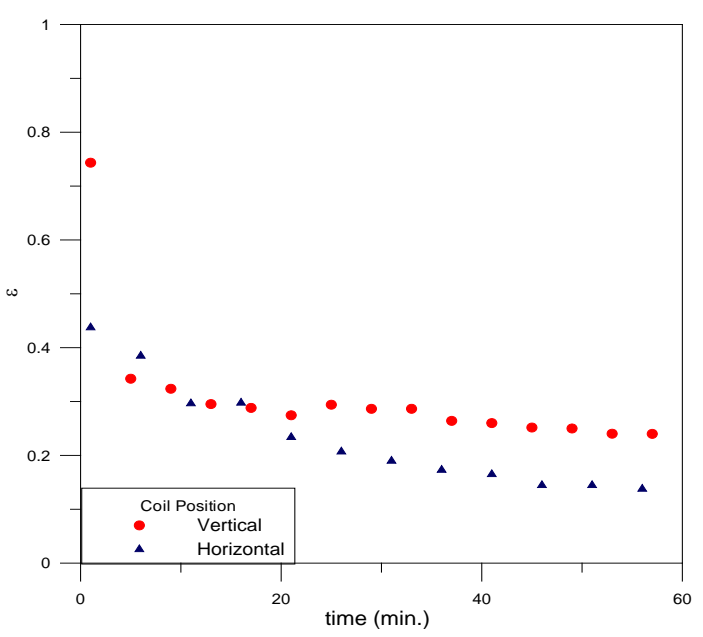

Figure 9. Comparison of efficiency between vertical vs. horizontal coil.

\subsection{Effect of mass flow rate}

The 10-looped vertical coil is then tested for two different flow rates to illustrate the influence of Reynolds Number on the performance of the coil. Figure 10 shows the theoretical 1-D analysis for the tube verses coil heat exchangers. It is clearly seen that the performance is slightly enhanced when Reynolds number increases, though more tests should be conducted at different $R e$ in order to support such conclusion. Figure 11, which illustrates the experimental results, also shows similar behavior of Re. This could be attributed to the fact that the Nusselt Number of the fluid carried by the coil improves as $R e$ increases; hence, enhancing the convective heat transfer with the medium in the container.

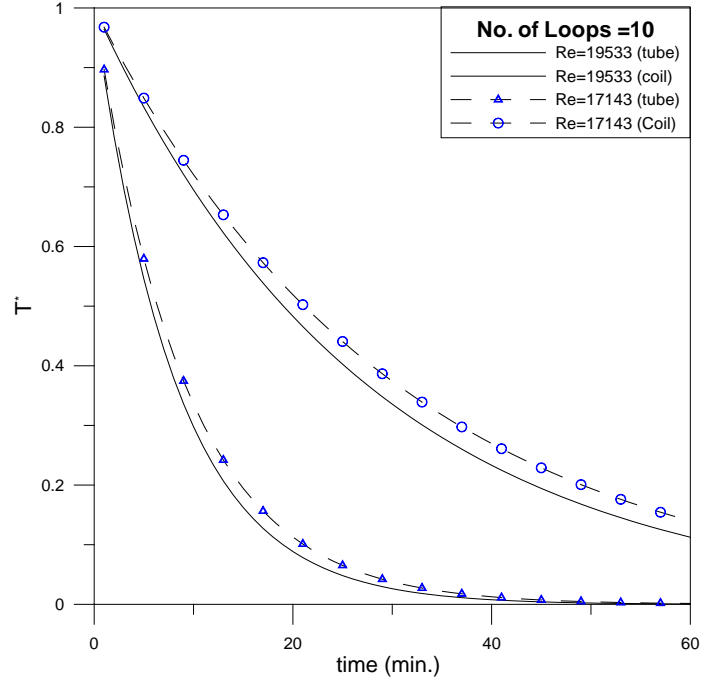

Figure 10. Effect of Reynolds number on the rate of heat transfer.

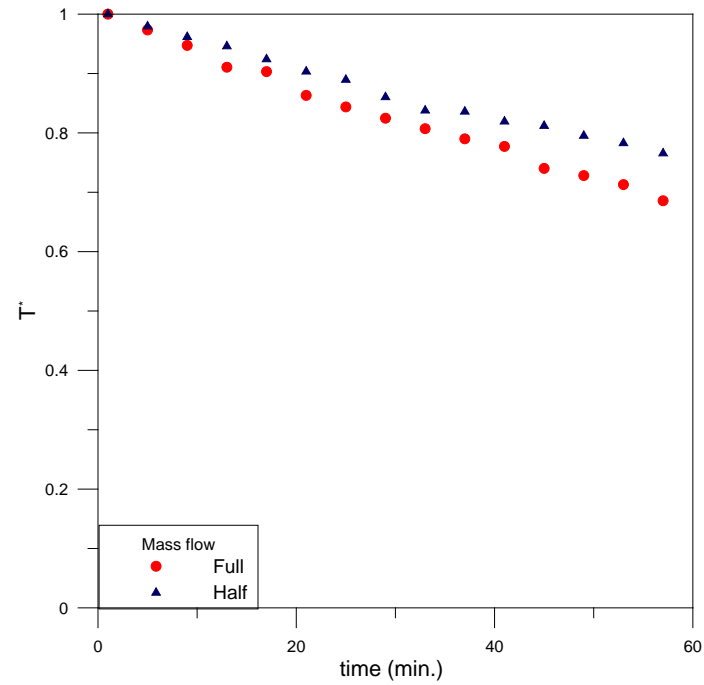

Figure 11. Coil performance for different mass flow.

\section{Uncertainty analysis}

Inconsistencies exit in experimental engineering analyses due to precision and accuracy measurements. The purpose of this section is to describe the uncertainty analyses of the sensors that were used during the implementation of the experimental tests.

The total outside heat transfer area for spiral coil is listed earlier in Eq. (5):

$A_{\text {coil }}=\pi D A_{\mathrm{o}} n(1+2 \pi \alpha n / D)$

where $A_{\mathrm{o}}$ stands for the heat transfer area per unit length $\left(\pi d_{\mathrm{p}}\right) ; n$ is number of turns; $D$ is helix diameter; and $\alpha$ is the rate of increase of radius, in $\left(\mathrm{m} \mathrm{rad}^{-1}\right)$. 
Spiral coil heat exchanger effectiveness can be written in the following form:

$\varepsilon=\frac{\rho \dot{Q} c_{\mathrm{p}} \Delta T}{h A_{\mathrm{t}} \mathrm{LMTD}} ;$ where LMTD $=\frac{2 T_{\mathrm{w}}+\Delta T}{\ln \frac{T_{\mathrm{w}}-T_{\mathrm{o}}}{T_{\mathrm{w}}-T_{\mathrm{i}}}}$.

For simplicity, we assume that the effectiveness does not have sensitivity to fluid properties such as density, viscosity, specific heat, and conductivity. However, it is sensitive to the convective heat transfer coefficient $(h)$, which in turn is influenced by the Reynolds number.

Hence, Eq. (11a) becomes:

$\varepsilon=\frac{\rho \dot{Q} c_{\mathrm{p}} \Delta T}{0.023 \operatorname{Re}^{0.8} \operatorname{Pr}^{0.4} \times A_{\mathrm{t}} \times \mathrm{LMTD}}$

or in terms of parameters' sensitivity:

$\varepsilon=\varepsilon\left(Q^{0.2}, D, d_{\mathrm{p}}^{0.8}, \Delta T, T_{\mathrm{w}}\right)$.

The independent variables, or the measured parameters, are: inlet and outlet flow temperatures and pressures $(T, p)$, helix diameter $(D)$, fluid flow rate $(Q)$, pipe diameter $\left(d_{\mathrm{p}}\right)$, and the conducting volume temperature $\left(T_{\mathrm{w}}\right)$. Hence, the uncertainty equation of the coil performance $(\varepsilon)$ is:

$$
\begin{aligned}
U_{\varepsilon} & =\left[\left(\frac{\partial U_{\varepsilon}}{\partial Q} \delta Q\right)^{2}+\left(\frac{\partial U_{\varepsilon}}{\partial D} \delta D\right)^{2}+\left(\frac{\partial U_{\varepsilon}}{\partial d_{\mathrm{p}}} \delta d_{\mathrm{p}}\right)^{2}\right. \\
& \left.+\left(\frac{\partial U_{\varepsilon}}{\partial(\Delta T)} \delta(\Delta T)\right)^{2}+\left(\frac{\partial U_{\varepsilon}}{\partial T_{\mathrm{w}}} \delta T_{\mathrm{w}}\right)^{2}\right]^{1 / 2}
\end{aligned}
$$

White (2003) elaborates the approach that was used in the analysis.

The precision of the flow-meter device used in the experiment is $1.5 \%$, the tolerance in the size of the pipe is $0.5 \%$, the deviation in the temperature sensors are within $0.1 \mathrm{~K}$, and that of the helix diameter is $2 \%$.

$$
\begin{aligned}
U_{\varepsilon} & =\left[(0.2 \times 1.5)^{2}+(1 \times 2)^{2}+(0.8 \times 0.5)^{2}+(1 \times 0.2)^{2}\right. \\
& \left.+(1 \times 0.1)^{2}\right]^{0.5} \\
U_{\varepsilon} & =2.16 \%
\end{aligned}
$$

The dominant parameter that caused considerable impact was the helix diameter.

\section{Conclusions}

Experimental studies on transient natural convection heat transfer from spiral coil are conducted. Different coils are embedded in a conducting volume container to study the transient behavior of heat transfer. Impact of coil geometry and orientation, number of loops, and Reynolds number are documented. Results have shown that coil geometry significantly influences the performance and efficiency of the heat transfer. The following summarize the outcomes:

1. Vertically-positioned coil was shown to be more effective than horizontally-positioned coil in terms of transferring heat. The enhancement was found to be doubled.

2. Although including the coil curvature during the theoretical analysis somewhat interprets the actual behavior of the coil, such analysis fails to predict the effect of coil orientation. This supports the demand of experimental investigation as an alternative robust method.

3. Doubling the number of loops per meter length of spiral coil enhances the rate of heat transfer by $\sim 130 \%$.

4. Increasing the Reynolds number also improves the performance of the coil. However, further experiments at different values should be conducted to support such conclusion. 


\section{Appendix A}

Consider a straight pipe with a length $(L)$ and diameter $(d)$ carrying a fluid with density $(\rho)$, specific heat $\left(C_{\mathrm{p}}\right)$, viscosity $(\mu)$, and thermal conductivity $(k)$. The pipe is surrounded by another fluid with different properties. The fluid inlet and exit temperatures are respectively $\left(T_{\mathrm{i}}\right)$ and $\left(T_{\mathrm{e}}\right)$ and the surrounding temperature is $(T \infty)$.

Using the energy balance law for the 1-D case:

$\dot{Q}=\dot{m} \times C_{\mathrm{p}} \times\left(T_{\mathrm{e}}-T_{\mathrm{i}}\right)=U \times A_{\mathrm{s}} \times \Delta T_{\text {ave }}$

where $U$ is the overall heat transfer coefficient, and could be determined from:

$\frac{1}{U A_{\mathrm{s}}}=\frac{1}{h_{\mathrm{i}} A_{\mathrm{i}}}+\frac{\ln \left(d_{\mathrm{po}} / d_{\mathrm{pi}}\right)}{2 \pi k L_{\mathrm{str} .}}+\frac{1}{h_{\mathrm{o}} A_{\mathrm{o}}}$.

$h_{\mathrm{i}}$ is the forced convective heat transfer coefficient of the fluid inside the pipe, that was obtained from the DittusBoelter equation for straight pipe (Yunus and Afshin, 2010):

$N u=0.023 \operatorname{Re}^{4 / 5} \operatorname{Pr}^{n} ; \quad n=0.4$ for heating;

$n=0.3$ for cooling.

$h_{\mathrm{o}}$ is the natural convective heat transfer coefficient of the fluid surrounding the pipe.

$\Delta T_{\text {ave }}$ is the average temperature difference, evaluated for simplicity, from the arithmetic mean.

$\Delta T_{\mathrm{ave}} \approx \frac{\left(T_{\infty}-T_{\mathrm{i}}\right)+\left(T_{\infty}-T_{\mathrm{e}}\right)}{2}$

The error in using the arithmetic mean instead of the logarithmic mean is less than $1 \%$ (Yunus and Afshin, 2010).

Combine A1 and A 3 then solve for $T_{\mathrm{e}}$ (exit temperature of the straight portion which is the inlet coil temperature $T_{\mathrm{ci}}$ ):

$T_{\mathrm{ci}}=T_{\mathrm{i}}\left(\frac{1-0.5 \mathrm{NTU}}{1+0.5 \mathrm{NTU}}\right)+T(t) \times\left(\frac{\mathrm{NTU}}{1+0.5 \mathrm{NTU}}\right)$.

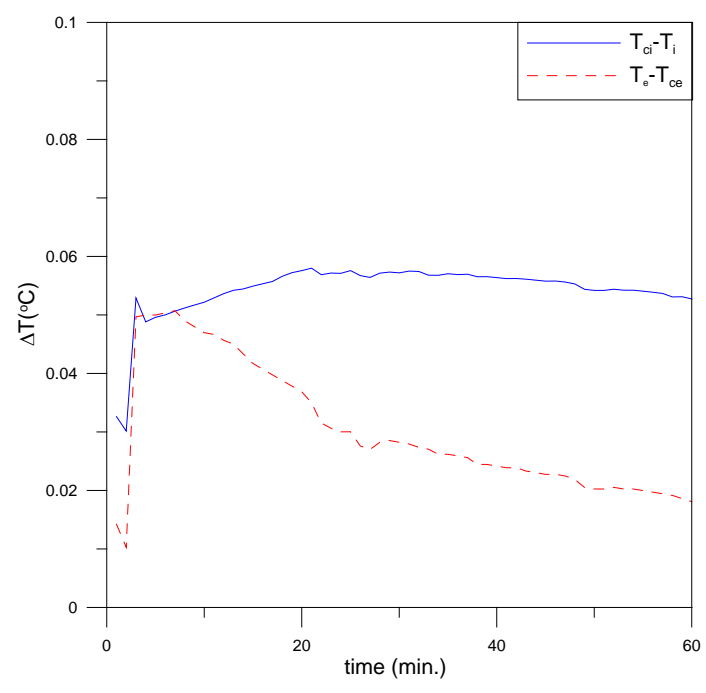

Figure A1. Temperature difference across the uncoiled portion.

Solve again for $T_{\mathrm{i}}$ (inlet temperature of the straight portion which is the outlet coil temperature $T_{\text {ce }}$ ):

$T_{\mathrm{ce}}=T_{\mathrm{e}}\left(\frac{1+0.5 \mathrm{NTU}}{1-0.5 \mathrm{NTU}}\right)-T(t) \times\left(\frac{\mathrm{NTU}}{1-0.5 \mathrm{NTU}}\right)$.

NTU in Eq. (A4) stands for number of transfer unit: $\mathrm{NTU}=\frac{U A_{\mathrm{s}}}{\dot{m} C_{\mathrm{p}}}$; and $T(t)$ is the transient temperature of the surrounding fluid.

Figure A1 illustrates the difference in temperature of the fluid inside the spiral coil, with time across the uncoiled portion at entrance and exit. The assumption of using measured temperatures as coil inlet and outlet temperatures can be easily justified by looking at the maximum temperature difference in the graph. The graph shows that the temperature difference did not even reach the precision of the temperature sensors fixed in then apparatus. 
Edited by: M. Teodorescu

Reviewed by: two anonymous referees

\section{References}

Alalaimi, M., Lorente, S., and Bejan, A.: Thermal coupling between a spiral pipe and a conducting volume, Int. J. Heat Mass Trans., 77, 202-207, 2014.

Ali, M.: Natural convection heat transfer from vertical helical coils in oil, Heat Trans. Eng., 27, 79-85, 2006.

Beigzadeh, R. and Rahimi, M.: Prediction of heat transfer and flow characteristics in helically coiled tubes using artificial neural network, Int. Commun. Heat Mass, 39, 1279-1285, 2012.

Dean, W. R.: Note on the motion of fluid in a curved pipe, Philos. Mag., 4, 208-223, 1927a.

Dean, W. R.: The stream line motion of fluid in a curved pipe, Philos. Mag., 5, 208-223, 1927b.

Elazm, M., Ragheb, A., Elsafty, A., and Teamah, M.: Numerical Investigation for the Heat Transfer Enhancement in Helical Cone Coils over Ordinary Helical Coils, J. Eng. Sci. Tech., 8.1, 1-15, 2013.

Elsayed, A., Dadah, R. A., Mahmoud, S., and Rezk, A.: Investigation of flow boiling heat transfer inside small diameter helically coiled tubes, Int. J. Refrig., 35, 2179-2187, 2012.

Freng, Y. M., Lin, W. C., and Chieng, C. C.: Numerically investigated effects of different Dean number and pitch size on flow and heat transfer characteristics in a helically coiled tube heat exchanger, Appl. Therm. Eng., 36, 378-385, 2012.

Gupta, R., Wanchoo, R. K., and Ali, T.: Laminar flow in helical coils a parametric study, Industrial and Engineering Chemistry Research, 50, 1150-1157, 2011.
Heo, J. and Chung, B.: Influence of helical tube dimensions on open channel natural convection heat transfer, Int. J. Heat Mass Trans., 55, 2829-2834, 2012.

Jamshidi, N., Farhadi, M., Ganji, D., and Sedighi, K.: Experimental analysis of heat transfer enhancement in shell and tube helical tube heat exchangers, Appl. Therm. Eng., 51, 644-652, 2013.

Sanner, B., Karytsas, C., Mendrinos, D., and Rybach, L.: Current status of ground source heat pumps and underground thermal energy storage in Europe, Geothermics, 32, 579-588, 2003.

Sasmito, A. P., Kurnia, J. C., and Mujumdar, A. S.: Numerical evaluation of laminar heat transfer enhancement in nanofluid flow in coiled square tubes, Nanoscale Res. Lett., 6.1, 1-14, 2011.

Seara, J. F., Pontevedra, C. P., and Dopazo, J. A.: On the performance of a vertical helical coil heat exchanger numerical model and experimental validation, Appl. Therm. Eng., 62, 680-689, 2014.

Srinivasan, P. S., Nandapurkar, S. S., and Holland, F. A.: Friction factor for coils, Trans. Inst. Chem. Eng., 48, 156-161, 1970.

White, F. M.: Fluid Mechanics, 5th Edn., McGraw-Hill Inc., New York, 2003.

Wijeysundera, N., Ho, J., and Rajasekar, S.: The Effectiveness of a Spiral Coil Heat Exchanger, Int. Commun. Heat Mass, 23, 623631, 1996.

Yunus, A. C. and Afshin, J. G.: Heat and Mass Transfer, 4th Edn., McGraw Hill, New York, USA, 2010.

Zhu, H., Hanqing, W., and Guangxiao, K.: Experimental study on the heat transfer enhancement by Dean Vortices in spiral tubes, Int. J. Energy Environ., 5, 317-326, 2014. 